Int. J. Dev. Biol. 61: 171-178 (2017)

doi: $10.1387 / \mathrm{ijdb} .160279 \mathrm{as}$

\title{
Building functional units of movement-generation and movement-sensation in the embryo
}

\author{
PELEG HASSON ${ }^{1}$, TALILA VOLK ${ }^{*, 2}$ and ADI SALZBERG ${ }^{*, 1}$ \\ ${ }^{1}$ Department of Genetics and Developmental Biology, The Rappaport Faculty of Medicine and Research Institute, \\ Technion-Israel Institute of Technology, Haifa and \\ ${ }^{2}$ Department of Molecular Genetics, Weizmann Institute of Science, Rehovot, Israel
}

\begin{abstract}
The musculoskeletal and proprioceptive sensory systems exhibit intricate crosstalk between force generation, force sensation and force transmission, all of which are critical for coordinated animal locomotion. Recent developmental studies of the musculoskeletal and proprioceptive units of the invertebrate Drosophila embryo, have revealed several common molecular and structural principles mediating the formation of each of these systems. These common principles, as well as the differences between the developmental programs of the two systems, are discussed. Interestingly, a molecular pathway triggered by the Neuregulin/Vein ligand-dependent activation of the epidermal growth factor receptor (EGFR) pathway, which upregulates the early growth response (EGR)-like transcription factor Stripe, is utilized not only by the Drosophila muscle-tendon and proprioceptive organ-ectoderm attachment, but also by their vertebrate counterparts. An additional theme that has been observed during the development of the musculoskeletal system in both invertebrates and vertebrates is the functional importance of the extracellular matrix and its adhesion receptors. The contribution of mechanical forces to proper junction formation between muscles and tendons and between the sensory cap/ligament cells and their epidermal attachment cells is discussed. The structural and genetic similarities between the musculoskeletal and the proprioceptive systems offer new perspectives as to their common developmental nature.
\end{abstract}

KEY WORDS: muscle, tendon, proprioceptor, chordotonal

\section{Assembling the musculoskeletal system}

The development of the musculoskeletal system is fundamental for the formation of motile multicellular organisms. Functional musculoskeleton consists of a contractile component - the muscle fibers, a connecting elastic cellular constituent - the tendons, and a rigid component - the cuticle (in invertebrates) or bone (in vertebrates) (Schnorrer and Dickson, 2004; Schweitzer et al., 2010). All three constituents are tightly linked through specialized junctional elements, which include adhesion receptors and various types of extracellular matrix components that connect these elements together and provide elastic properties to the system (Subramanian and Schilling, 2014; Subramanian and Schilling, 2015). How these distinct elements assemble during embryonic development is the subject of an active research in both invertebrates and vertebrates species. Below is a brief summary of the strategies taken by the invertebrate model organism Drosophila melanogaster as well as zebrafish and mouse as the vertebrate representatives for musculoskeletal assembly; similarities and differences between these two model systems will be highlighted. In this review we will primarily focus on mechanisms that regulate the interactions between muscles and tendons rather than bones and tendons (for a specific review on this junction see (Lu and Thomopoulos, 2013; Zelzer et al., 2014).

\section{Building a functional proprioceptor}

Coordinated locomotion depends on fully functional musculoskeletal system, but it also requires a sensory system that conveys information to the brain about the posture and movement of body

Abbreviations used in this paper: $\mathrm{ChO}$, chordotonal organ; EGR, early growth response; EGFR, epidermal growth factor receptor; MTJ, myotendinuous junction.

\footnotetext{
*Address correspondence to: Adi Salzberg. Department of Genetics and Developmental Biology, Rappaport Faculty of Medicine, Technion, P.O.Box 9649, Haifa 3109601, Israel. Tel: (+972)-4-8295455 Fax: (+972)-4-8295225 - e-mail: adis@technion.ac.il - iD http://orcid.org/0000-0002-0427-9809 or Talila Volk. Department of Molecular Genetics, Weizmann Institute of Science, Rehovot 76100, Israel. Tel: 972-8-9342426. Fax: 972-8-9344108. (iD http://orcid.org/0000-0002-3800-2621
}

Accepted: 10 August, 2016.

ISSN: Online 1696-3547, Print 0214-6282 
parts. This sensory system, referred to as the proprioceptive system, is patterned in coordination with the musculoskeletal system and shares some of the underlying patterning mechanisms.

In vertebrates, the most prominent type of muscle stretch proprioceptors consists of la-afferent neurons that innervate specialized encapsulated intrafusal muscle fibers (muscle spindles) and relay information about the muscle contractile status directly to the central nervous system (Crowe and Matthews, 1964). The muscle spindles are also innervated by fusimotor efferent nerves, which are required to maintain the stretch sensitivity of the spindle as the muscle contracts or stretches. Muscle spindles sense alterations in muscle fiber length, but they cannot sense changes in the length of the muscle-tendon complex that stem from tendon stretching. Another type of mechanosensory organs, the Golgi tendon organs, which are innervated by lb-afferent neurons, provide the complementary ability to sense the tension of tendons (reviewed in (Jami, 1992), however their function is less understood.

Although invertebrate proprioceptors are structured very differently from vertebrate proprioceptors, the underlying principle of action is similar. In both systems a proprioceptive neuron responds to relative displacement of two anchoring points whose distance from each other changes as a result of muscle contraction. In invertebrates however, (e.g. Drosophila) the proprioceptive neurons do not innervate the muscle itself; rather, they innervate sensory structures that are attached to the cuticle, and sense muscle contractions indirectly by sensing conformational changes exerted on the exoskeleton by muscle contractions.

The major proprioceptive system in Drosophilaconsists of stretchreceptive organs called chordotonal organs (ChO) (Caldwell et al., 2003), which are stretched sub-epidermally in close proximity to the layer of body-wall muscles (the fly larva equivalent of skeletal muscles) (Figure 1). They attach to the cuticle via specialized epidermal attachment cells and are activated by relative displacement of the two attachment points that leads to elicitation of a neuronal impulse (Reviewed in Field and Matheson, 1998).

There are several subtypes of ChOs, but they all contain a sensory unit that consists of a bipolar neuron enwrapped by a scolopale cell. The scolopale cell is attached on both its 'dendritic' side and 'axonal' side to accessory cells (cap and ligament cells, respectively) that attach the organ to its epidermal attachment cells (Figure 1). Thus, similarly to the musculoskeletal system, the proprioceptive system in Drosophila consists of three elements: a sensory unit that translates mechanical stimuli into neuronal signals, the attachment cells that connect the sensory unit to the cuticle, and the cuticle itself. Similarly to the musculoskeletal system the three components of the proprioceptive system are tightly linked through specialized junctional components required for the maintenance of organ integrity and function. Many of the proteins characterizing the myotendinuous junction (MTJ), including specific adhesion receptors and extracellular matrix components, are shared by the ChO junction (Greenblatt Ben-El et al., 2017). As discussed below, some of the molecular mechanisms involved in the assembly of the musculoskeletal system are also involved in the assembly of functional proprioceptors.

\section{Muscle targeting to tendons in Drosophila: a combination of intrinsic and extrinsic signaling}

Similarly to $\mathrm{ChO}$, muscle fibers are born distant from the final attachment site. What are the mechanisms directing the precise match between muscle fibers and tendons? Several experimental evidences exclude specific one-to-one relationships between a given muscle and a given tendon, and support an alternative strategy by which distinct muscle types are capable of binding to various tendons located at different ectodermal sites. For example, transforming the identity of the thoracic muscles into abdominal muscles did not affect their ability to attach thoracic tendons (Greig and Akam, 1993). Consistently, the key transcription factor regulating tendon cell identity, Stripe, an EGR-like zinc finger protein, is common to all future tendon cells in the embryo, and so far tendons with specific identity were not described (Frommer et al., 1996; Volk and VijayRaghavan, 1994). Overexpression of Stripe in the entire ectoderm promotes ectopic adhesion of muscles without any sign of specificity, implying that once the tendon cell fate has been defined it will bind with any muscle type (Gilsohn and Volk, 2010a; Gilsohn and Volk, 2010b).
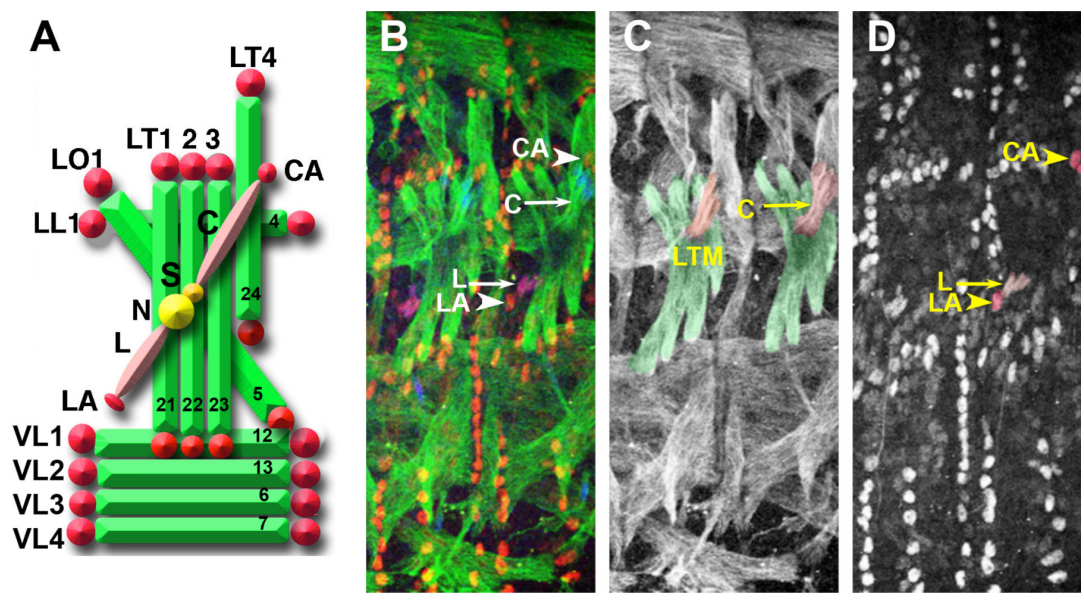

Fig. 1. The proprioceptive chordotonal organs develop in close proximity to the body-wall muscles and tendons in Drosophila embryos. (A) Schematic representation of a lateral chordotonal organ (ChO) stretched across the group of lateral transverse (LT) muscles in an abdominal segment of a mature Drosophila embryo. For the sake of simplicity, only one of the eight ChOs present in each abdominal segment is shown. Similarly, only ten of the 30 body-wall muscles present in each of the segments are depicted in the scheme (green bars) with their corresponding tendons (red circles). The numbers denote the specific muscle identity. LT1-4, lateral transverse 1-4; LO1, lateral oblique 1; LL 1, lateral longitudinal 1; VL1-4, ventral longitudinal 1-4. The ChO consists of a neuron enwrapped by a scolopale cell lyellow circles marked as $N$ and $S$, respectively), two accessory cells, the cap cell on the dendritic side and the ligament cell on the axonal side of the sensory unit (pink oval shapes marked as $C$ and L, respectively) and two attachment cells (depicted in red) that attach the organ to the cuticle, the cap-attachment (CA) and ligament attachment (LA) cells. (B) An abdominal segment of a mature Drosophila embryo immune-stained for beta3-Tubulin (green) and Stripe (red), demonstrating the pattern of muscles, tendons and ChOs. (B) The anti-beta3-Tubulin staining is shown separately. The lateral transverse muscles (LTM) are highlighted in green; the cap cells of the LCh5 organs (C) are highlighted in pink. (C) The anti-Stripe staining is shown separately. The ligament cells (L) of the LCh5 organ are highlighted in pink; the cap-attachment (CA) and ligament-attachment (LA) cells are highlighted in red. 
Muscles on the other hand show high degree of fate specificity. Each of the 30 types of muscle fibers present in a single abdominal embryonic hemisegment expresses a unique combinatorial set of transcription factors dictating muscle directionality, length, and number of nuclei to be fused (Baylies et al., 1998; Schejter and Baylies, 2010). These and other observations support the notion that the specificity of the muscle-tendon interaction is primarily dictated by the type of muscle rather than the identity of the tendon. Moreover, muscles contribute a differentiation factor to tendons following muscle-tendon encounter. This signal is Vein/Neuregulin, the only secreted ligand of the single EGF receptor (EGFR) present in the Drosophila genome. Vein/Neuregulin secretion from muscles leads to non-autonomous activation of the EGFR/MAPK signaling in the closest tendon cell, essential for further maintenance of Stripe expression in the muscle-bound tendon cell (Yarnitzky et al., 1997). Thus, whereas Stripe potentiates ectodermal cells to become tendons, a muscle-dependent activation of the MAPK signaling in these ectodermal cells is further required for determination of the tendon cell fate. In addition to the positive feedback by MAPK signaling at the transcriptional level of stripe, it also provides a positive post translational signal as a result of a direct phosphorylation of the hnRNP K RNA binding protein HOW/Quaking, whose binding activity to the mRNA of the stripeA isoform promotes its stabilization, leading to the elevation in the levels of StripeA required for tendon cell maturation (Lejard et al., 2011; Nabel-Rosen et al., 1999; Nabel-Rosen et al., 2002; Nir et al., 2012; Volohonsky et al., 2007). This muscle-to-tendon positive feedback forms the basis for a muscle-dependent tendon cell differentiation and highlights the key role of muscle binding in tendon cell differentiation.

\section{Targeting muscles to tendons and formation of the myotendinuous junction in vertebrates}

The mechanisms that regulate tendon and MTJ formation in vertebrates are still largely unclear essentially due to the lack of molecular tools and markers that mark progenitors of these tissues. Although as in Drosophila, EGR transcription factors, EGR-1 and EGR-2, are expressed in tendons, they do not regulate tendon identity but rather promote their differentiation (Guerquin et al., 2013; Lejard et al., 2011). The finding that Scleraxis (Scx), a bHLH transcription factor, marks tendon progenitors (Schweitzer et al., 2001) allowed studying the early stages of tendon development and consequently its interactions with the neighboring tissues - muscles and bones. However, deletion of scx although resulting in tendon deformities, did not lead to loss of tendon identity or MTJ formation, suggesting that other factors may be responsible for tendon identity, or that it is regulated via a combination of factors (Murchison et al., 2007).

The first interaction between muscles and tendon progenitors occurs in the somites where elongating myofibers anchor along the somitic boundaries. These intersomitic regions, also called myosepta, are not bona fide tendons as they do not connect the muscle to a bone. Functionally however, they act as tendons by transmitting muscle contractile forces. Because many of the processes and proteins participating in anchoring of the somitic myofibers to the myosepta are similar to those taking place at later stages we shall refer to these as MTJs.

Key to our understanding of MTJ formation is the need to form an intermediate region that connects two mechanically different tissues - the muscle and the tendon. To overcome the biophysical differences between muscles and tendons, the MTJ is a region with distinct extracellular constituents that grant its unique mechanical properties and allows the attachment of the myofibers onto the tendon's matrix. Thus, major players of MTJ formation are extracellular components such as Thrombospondin (Tsp), Laminin and Fibronectin (FN) that apart from regulating matrix biomechanics also act as integrin ligands and as such facilitate the anchoring of the integrin expressing myofibers to the tendon matrix. In contrast, the dystroglycan complex (DGC) that is involved in myofiber anchoring to the ECM and also at the MTJ is not required for initial MTJ formation (Snow and Henry, 2009).

In zebrafish, slow and fast myofibers are spatially segregated within the somite. Slow fibers initially reside in the medial part of the somite and migrate laterally in a process culminating in fast fibers being in a medial position (Devoto et al., 1996). Notably, slow and fast fibers adhere to distinct ECM molecules located along the somitic boundaries. Unlike Laminin which is expressed throughout the MTJ, FN distribution is initially observed medially along the slow fiber anchoring sites; coinciding with slow fiber lateral migration, in a laminin-MMP11 dependent mechanism, FN is lost in the medial domain and accumulates laterally (Jenkins et al., 2016; Snow and Henry, 2009). Because FN is not observed at the fast fiber anchoring sites it is suggested that the MTJs consists of distinct microdomains depending on fiber type. Thus, similarly to Drosophila where muscle identity promotes tendon differentiation, in zebrafish the fiber type controls the type of MTJ molecules.

Similarly to Drosophila, interactions between the distinctelements of the musculoskeletal system are critical for maintenance of the MTJ in later stages of musculoskeletal development. Chick in ovo manipulations demonstrated that development of the muscle and tendon lineages is independent of each other in early developmental stages, yet at a later stage interdependence is observed (Kardon, 1998). Work by Schweitzer and colleagues recently showed that in the absence of limb muscles, zeugopod tendons are induced but are not maintained and do not further differentiate, leading to their loss. In contrast, autopod tendons, although smaller than wild-type, are present and differentiate normally in these muscle-less limbs. These latter tendons, however, rely on cartilage condensation for their induction (Huang et al., 2015). Fgf4 represents a candidate secreted signal to carry out the crosstalk between muscles and tendons in vertebrates, similarly to the Drosophila EGFR ligand Vein. Fgf4 secreted from muscle tips is required for maintaining the tendon differentiation factors Scx and Tenascin C (EdomVovard et al., 2002). Feedback mechanisms such as the latter in which tendon differentiation relies on muscle binding might explain how even when limb muscles are mispatterned, MTJs are not affected and muscles are still bound to tendons albeit inserted the wrong locations (DeLaurier et al., 2006; Duboc and Logan, 2011; Hasson et al., 2010). Altogether, these mechanisms ensure an orchestration and synchronization of the development of the distinct musculoskeletal units required for the generation of a functional 'movement-generation' unit.

\section{Attachment of chordotonal organs to ectodermal attachment cells: a combination of cell-autonomous and non-autonomous inductive processes}

The contractile system (muscles and tendons) and the proprioceptive system (ChO and their attachment cells) constitute two parts 
of a single system that controls animal locomotion. Although the ChOs develop independently of the body wall muscles, these two systems must be patterned in a coordinated manner to guarantee accurate readout of muscle contractions and its translation into proprioceptive neuronal signals. The mechanisms responsible for the accurate matching between the patterning of muscles and proprioceptors in the fly are not fully understood. However, many lines of similarity can be drawn between the patterning of the muscle-tendon functional unit and that of the ChO and their specialized attachment cells.

Similarly to a muscle, which needs to be stably anchored on both its ends to immobile tendon cells in order to translate its contraction into movement of different body parts, a ChO must be anchored on both its sides to stationary epidermal cells in order to sense body movement. In addition, similarly to muscles that exhibit intrinsic directional elongation, the migrating ligament and cap cells of the ChOs do not depend on their epidermal attachment cells for elongation and migration, but rather are required for proper differentiation of the latter. The best-studied example is that of the most prominent larval $\mathrm{ChO}$, the pentascolopidial organ (LCh5). At one edge of the organ, the cap cells attach to the cuticle via lineage related epidermal attachment cells (Brewster and Bodmer, 1995). At the other edge of the organ the ligament cells migrate, pulling and stretching the organ, from a posterior dorsal region of the segment to an anterior lateral region of the segment (Inbal et al., 2003; Kraut and Zinn, 2004). Upon reaching their destination, the ligament cells secrete Vein, which activates the EGFR in the epidermal cell they contact. Activation of EGFR signaling leads to up-regulation of StripeB that induces the differentiation of a functional ligament-attachment cell (Inbal et al., 2004).

Although Stripe is not involved in the initial steps of capattachment cell development, it is required for later aspects of cell differentiation and maturation (Inbal et al., 2004). Similarly to the switch from StripeB to StripeA expression that takes place during tendon cell maturation, StripeAexpression becomes evident in the $\mathrm{ChO}$ attachment cells in late embryogenesis during the maturation of the

Fig. 2. Stripe-dependent patterning of the contractile and proprioceptive systems in Drosophila: variation on a common theme. The scheme shows three phases in the embryonic patterning of the muscle-tendon system, on the left, and that of the chordotonal organ (ChO) on the right. The first phase consists of muscle and ChO ligament cell extension towards their prospective epidermal attachment site. The pre-tendon cell expresses the EGR protein StripeB $(S r B)$ prior to muscle attachment, whereas the prospective ligament-attachment cell is not identifiable at this stage. Upon reaching their destination, both the muscle and the ligament cell secrete Vein/Neuregulin, which activates the EGFR signaling in the epidermal cells they contact. This leads to up-regulation of SrB transcription and tendon/Cho-attachment cell differentiation. The contact between the cells leads to migration arrest of the muscle or ligament cells, upregulation of the SrA isoform and the formation of a functional myotendinous or a ChO-attachment integrin-mediated junction between the cells.
ChO-attachment cells (Klein et al., 2010).

In mutant Drosophila embryos in which cell identities across the segment are altered due to mutations in segment polarity genes (e.g. hedgehog), thus altering the epidermal 'competence map', the ligament cells can still migrate to the right region, secrete Vein and recruit an attachment cell from within the nearest group of competent cells (Klein et al., 2010). The ability of the ligament cells to turn towards the miss-localized Stripe-expressing attachment cell suggests that this cell signals back to the ligament cells helping to shape the junction between them.

The fact that the same cassette of genes that underlie the crosstalk and mutual influence between muscles and tendons, namely Vein, EGFR and Stripe, also functions in the patterning of the ChOs and their attachment cells, suggests a generalized model for Stripe-dependent patterning of organs that depend on reciprocal interactions between cells from different lineages (Figure 2). Indeed, the same signaling cascade is also involved in the patterning and differentiation of vertebrate muscle spindles. It was shown in mice that the proprioceptive afferent nerve endings express Neuregulin1 (Nrg1), a mammalian Vein homolog. Nrg1 is then cleaved by the Bace1 (a protease beta-secretase) and activates the ErbB receptor in the intrafusal muscle fibers it contacts. This activation leads to the up-regulation of Egr3 expression (a mammalian Stripe homolog), and consequent muscle spindle differentiation (Hippenmeyer et al., 2002; Cheret et al., 2013). Egr3 plays an autonomous role in the intrafusal muscle fibers promoting their differentiation and fusimotor innervation by regulating gene expression (Oliveira Fernandes and Tourtellotte, 2015). The vertebrate proprioceptors are highly diverse, but very little is known about the mechanisms regulating their specification (Arber, 2012). Jessel and colleagues have recently shown that proprioceptors

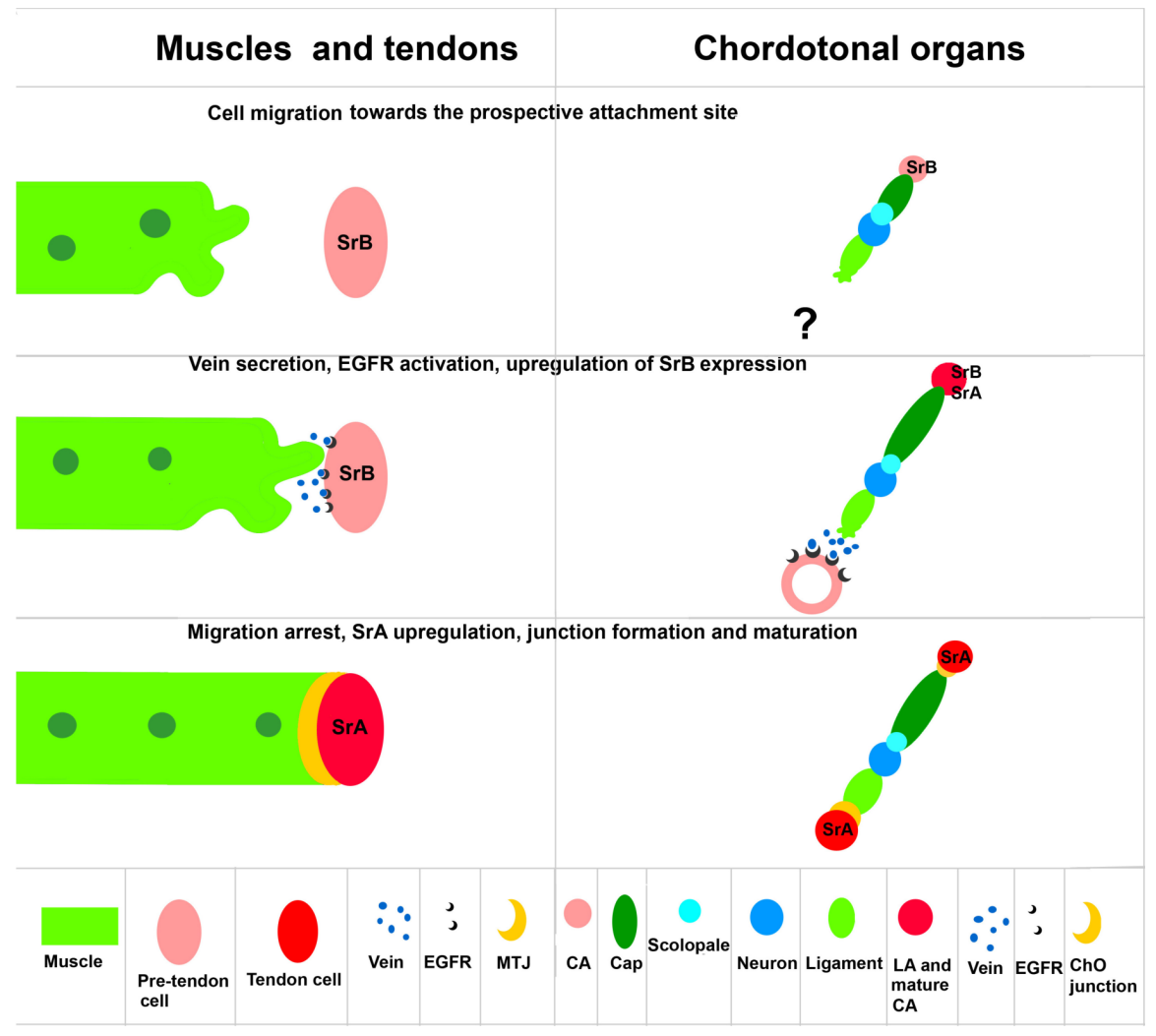




\section{wild-type}

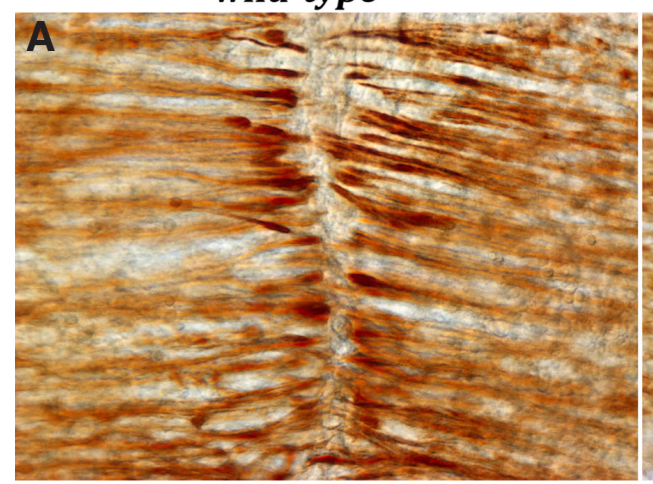

$\operatorname{LoxL} 3^{\%}$

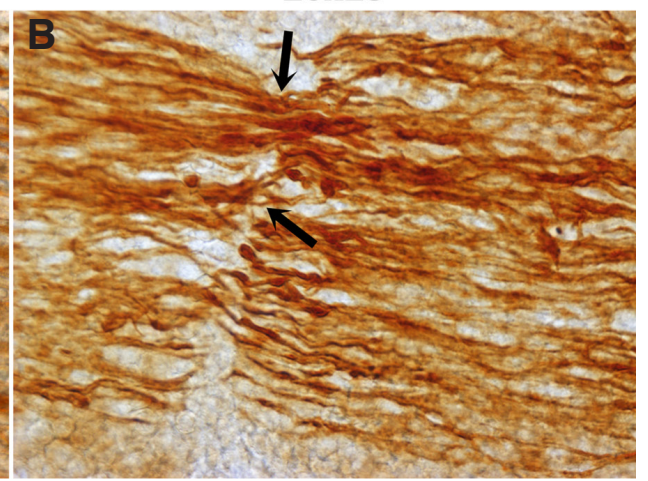

Fig. 3. LoxL3 regulates myofiber anchorage along the somitic boundary. Whole mount immunostaining for myosin heavy chain of E10.5 somites demonstrates that while in wild-type embryos (A) myofibers anchor along the somitic boundary, in LoxL3 mutants, in which the composition of the ECM changes (B) myofibers overshoot and anchor ectopically along the adjacent somite (black arrows).

has been shown to modulate Tsp and Integrin-beta1 accumulation at the muscle membrane, following muscle-tendon interaction (Gilsohn and Volk, 2010b). Additional tendon-specific proteins have been identified, including the adhesion receptors Nephrin/Hibris (Dworak et al., 2001), Unc-5 (Keleman and Dickson, 2001), Leucine-Rich Tendon specific protein, LRT (Wayburn and Volk, 2009), and Syndecan (Steigemann etal., 2004). Tendon-secreted proteins, including $\mathrm{Ne}$ trin A, Semaphorin-5C, Collagen XVIII/ Multiplexin, have been also described (Gilsohn and Volk, 2010a). Phenotypic analyses indicated that they do not function as active attractive cues, but rather as signals required for the arrest of muscle elongation.

acquire their muscle type identity in response to positional cues originating in the limb mesenchyme (Poliak et al., 2016).

\section{Tendons are essential for providing the stop signal, but} not for attractive cues, in Drosophila and in vertebrates

Live imaging of Drosophila muscle elongation towards tendons in embryos has established that distinct muscles exhibit intrinsic elongation polarity independently of tendons (Ordan et al., 2015). In the absence of tendons muscles do not lose their initial directional extension (Krzemien et al., 2012). It appears therefore that tendons and their specific surface receptors, or secreted signals, are essential for arresting muscle elongation and promoting muscle adhesion.

An essential, evolutionary conserved signal secreted from tendons is Tsp, an ECM component, which binds to the alpha2beta1 integrin specific muscle adhesion receptor (Subramanian and Schilling, 2014; Subramanian et al., 2007). Tsp represents the earliest tendon-specific secreted signal, essential for muscletendon adhesion. In its absence, most of the muscles fail to bind to tendons, but are still capable of binding to their neighboring muscles. Tsp promotes integrin accumulation at the muscle leading edge, which further leads to the arrest of muscle elongation and the initiation of MTJ formation. Interestingly, during larval stages Tsp does not appear to be maintained in the MTJ and is detected in vesicular-like particles in the muscle cytoplasm, possibly involved in protein translocation (Volk, T. personal observations). Tsp also plays an essential role in the attachment of $\mathrm{ChO}$ to their attachment cells and is required for proper localization of integrin in the cap cells (Greenblatt Ben-El et al., 2017). Although the stop signal for migrating $\mathrm{ChO}$ ligament or cap cells has not been identified, we have evidence demonstrating that the ligament-attachment cell is critical for stopping the migrating ligament cells of the LCh5 organ and later on for stopping the migration of the ventral VChB cap cell (Halachmi et al., 2016; Hassan et al., unpublished data). In addition, phenotypic analysis of tsp mutant embryos shows defects in ChO migration and a failure of the ventral ChO (VChB) to stall when they reach the ventral lateral transverse tendon cells (Greenblatt Ben-El et al., 2017).

The EGF-like-7 protein Slowdown, secreted from the tendons
The Slit/Robo signaling pathway had been recently implicated in the process of muscle elongation towards tendons. Slit is secreted by tendon cells, whereas muscle-specific Robo and Robo3 receptors respond on the muscle side to Slit (Ordan and Volk, 2015a). At the tendon side Slit undergoes cleavage into the $\mathrm{N}$-terminal active Slit-N, which is extremely stable, and in addition undergoes oligomerization, and associates with the tendon cell surfaces. The C-terminal Slit-C, is rapidly degraded. Slit cleavage is an important process because it leads to immobilization of the Slit signaling on tendons, restricting Slit into a short-range repulsion cue, which affect only the closest muscle inducing the arrest of its further elongation (Ordan et al., 2015). Robo2, (but not Robo and Robo3) is co-expressed with Slit by the tendon cells and has a non-signaling role in Slit tethering to the membrane of the tendon cell. Both Robo2-dependent Slit cleavage, and the formation of Slit-N oligomers on the tendon cell surfaces are critical for direct arrest of muscle elongation and for providing a stop signal for the approaching muscle, through binding to Robo and Robo3 receptors expressed by the muscles (Ordan and Volk, 2015b).

A highly similar process has evolved in vertebrate MTJ formation. Similarly to flies, no attractive cue secreted by the tendon has been found. It thus seems, that as observed in the fly, the tendon provides a site for muscle anchoring. While Tsp is expressed in vertebrate tendons (myosepta) it does not seem to play an early role in MTJ initiation as in flies. Rather, Tsp facilitates the organization of a stable matrix at the MTJ required for its maintenance (Subramanian and Schilling, 2014). In mice, this early role may have been taken by FN, an ECM structural glycoprotein that like Tsp serves as an integrin ligand. In both zebrafish and mouse, FN is required for somite formation and its knockdown or genetic deletion results in somite and somitic boundary abnormalities (George et al., 1993; Snow et al., 2008). At these stages, no subdivision to slow and fast fibers occurs in mice and FN does not shift along the medio-lateral axis of the MTJ. We have recently found that Lysyloxidase Like 3 (LoxL3), an ECM modifying enzyme is secreted by the elongating myofiber tip and oxidizes $\mathrm{FN}$ upon reaching to the MTJ. This oxidation activates integrin signaling within the myofibers facilitating their anchoring along the MTJ leading to the generation of an organized FN matrix (Kraft-Sheleg et al., 2016). Myofibers mutant for LoxL3 overshoot their anchoring site at the 
MTJ and attach to the adjacent muscle (somite) in a similar manner to that occurring in Drosophila Tsp mutants, suggesting that also in vertebrates tendons act as a stop signal to the growing myofiber (Figure 3). Altogether, formation of a stable MTJ relies not only on the ECM and adhesion molecules presented on the 'tendon side' but importantly on signals emanating from the muscle that modify the ECM into a stable matrix. These processes rely on integrin signaling during all stages of MTJ formation and maintenance on both muscle and tendon sides, a requirement conserved between vertebrates and invertebrates.

\section{Force transmitting mechanisms are involved in the maturation of tendons and muscles}

Mechanical force has been recently implicated as a key factor in promoting morphogenetic processes during embryonic development. Once the musculoskeleton connections and the MTJ have been established in late stages of embryonic development and muscle contraction begins, force is applied on both the tendon and the muscle sides, further shaping muscle and tendon maturation.

On the tendon side, dense array of polarized microtubule (MT) are detected in the direction of the contraction axis, where the plus end MT binding protein, EB1, is observed at the MTJ and MT begin to form parallel array connecting the MTJ to the cuticle. Shot/ACF7 has a major role in recruiting EB1 to the MTJ, thereby stabilizing microtubule plus ends at this site. In the absence of Shot/ACF7 in tendons, the arrangement and polarization of the MT is disrupted, leading to tearing of the tendons, abrogation of contractile force transmission, and eventually inability of the larva to move (Subramanian et al., 2003).

At the muscle side, force transmission is also essential for muscle maturation. It has been shown that manual disruption of force transmission between the tendon and the muscle by laser ablation of the MTJ, disrupts normal development of sarcomeres in the affected muscle (Weitkunat et al., 2014). Consistently, we recently found that Shot/ACF7 and EB1 mediate MT polarized organization at the muscle side as well. Knocking down Shot/ ACF7 specifically in muscles led to disruption of the MT pattern and resulted in aberrant sarcomere organization, as well as disruption of the myonuclear morphology (Wang et al., 2015). It appears therefore that the mechanical force developed between tendons and muscles following the formation of the MTJ, represents an essential signal for both the tendon and the muscle to undergo terminal differentiation.

Not much is known about the cell biological processes that take place upon force generation following MTJ formation in vertebrates. Using the muscular dysgenesis mice (mdg) in which due to a mutation in a voltage gated ion channel muscles do not contract, it was shown that the muscle contraction force is required for autopod tendon growth and for zeugopod tendon individuation (Huang et al., 2015).

\section{Force transmitting mechanisms and their developmental role in Drosophila proprioceptors}

The ChOs experience mechanical strains as a result of muscle contractions. However, even without muscle contractions, larval growth on its own imposes significant mechanical load on the ChO that remain attached to the cuticle on both their edges as the larva grows. Within three days of larval development the LCh5 organ increases its length approximately 5 -fold. During this period, the ChO attachment cells grow immensely and extend numerous MTrich extensions that might increase the contact area between the attachment cell and the substrate to which it attaches (Halachmi et al., 2016). It is assumed that the growing mechanical stresses are involved in inducing $\mathrm{ChO}$-attachment cell growth, but how the correlation between the two processes is achieved is still a mystery.

The ChO attachment cells share many properties with tendon cells. Both types of cells are defined by the expression of the transcription factor Stripe, they both require the bHLH transcription factor Delilah (Dei) for terminal differentiation, and both exhibit an elaborate MT-network. The connection between the ChO's cap and ligament cells to their corresponding epidermal attachment cells involves junctional elements similar to those used to connect muscles to tendons, such as integrin adhesion receptors, Shot and Tsp (Greenblatt Ben-El at al., 2017). This similarity reflects their function in force transmission. Of note is a unique isoform of alpha-Tubulin, alpha-Tub85E, expressed exclusively in the ChO and their attachment cells, possibly reflecting a difference between the strains sensed by the $\mathrm{ChO}$ attachment cells versus the strains sensed by tendon cells.

The fact that the identity and differentiation of both types of attachment cells (tendons and $\mathrm{ChO}$ attachment) is determined by Stripe expression, raises the question as to what distinguishes between these two developmental programs. Although a specific cell-identity regulator has not been identified, ectopic expression of Stripe in the ectoderm reveals domains that are competent to differentiate as tendon cells (expressing the common attachment cell markers, but are devoid of alphaTub85E; Klein et al., 2010) and domains that are competent to differentiate as $\mathrm{ChO}$ attachment cells (expressing the common attachment cell markers as well as alpha-Tub85E). This observation suggests that the epidermis is not equipotent in its response to Stripe expression and that the competence of cells to acquire one of the two attachment cell-identities (muscle attachment or $\mathrm{ChO}$ attachment) is embedded within the pre-patterned ectoderm and depends on segment polarity pre-pattern genes such as $h h$, en and $w g$. The division of the epidermis to mutually exclusive domains of alternative cell fates (tendons vs ChO attachment cells) may play a role in the copatterning of the two complementary systems. This is reminiscent of the patterning mechanism of the adult thorax in which a mutual exclusion of sensory bristles and tendons on the notum of the adult fly had been described (Usui et al., 2004).

\section{Perspectives}

While the signals and genes implicated in the formation of each cell type within the musculoskeletal system are slowly unraveled, understanding the mechanisms underlying the crosstalk between these tissues and the contribution of the mechanical load to the system are still unclear. Multiple lines of evidence, in both flies and vertebrates, demonstrate that the interactions between muscles and tendons, or between $\mathrm{ChO}$ and the cuticle, rely on modulation of ECM components that promote activation of integrin signaling in both directions (outside-in and inside-out) at the anchoring site. The extent of integrin signaling might be also affected by the mechanical load and by the composition of the extracellular matrix, however the molecular basis for this is unclear. 
The formation of the MTJ or the ChO-epidermis interactions induces the secretion of survival or differentiation factors (e.g., Vein in Drosophila and FGF4 in vertebrates). Surprisingly, only a handful of secreted factors that are functionally linked to the crosstalk between these tissues have thus far been identified. Uncovering additional growth factors in that context should promote a more comprehensive molecular understanding of the dynamic nature of these interactions.

Altogether, a gap still exists between our understanding of the mechanisms involved in the development of the distinct tissuespecific elements and the molecular knowledge of how they are integrated into a functional unit. Future work with a more holistic perspective and molecular analysis in the frame of whole organ development will allow closing this gap.

\section{References}

ARBER S (2012). Motor circuits in action: specification, connectivity, and function. Neuron 74: 975-989.

BAYLIES MK, BATE M, GOMEZ MR (1998). Myogenesis: A View from Drosophila. Cell 93: 921-927.

BREWSTER R, BODMER R(1995). Origin and specification of type ll sensory neurons in Drosophila. Development 121: 2923-2936.

CALDWELL JC, MILLER MM, WING S, SOLL DR, EBERL DF (2003). Dynamic analysis of larval locomotion in Drosophila chordotonal organ mutants. Proc Natl Acad Sci USA 100: 16053-10658.

CHERET C, WILLEM M, FRICKER FR, WENDE H, WULF-GOLDENBERG A, TAHIROVIC S, NAVE K-A, SAFTIG P, HAASS C, GARRATT AN, BENNETT DL, BIRCHMEIER C (2013). Bace1 and Neuregulin-1 cooperate to control formation and maintenance of muscle spindles. EMBO J 32: 2015-2028.

CROWE A, MATTHEWS PB (1964). The effects of stimulation of static and dynamic fusimotor fibres on the response to stretching of the primary endings of muscle spindles. J Physiol 174: 109-131.

DELAURIERA, SCHWEITZERR, LOGANM (2006). Pitx1 determines the morphology of muscle, tendon, and bones of the hindlimb. Dev Biol 299: 22-34.

DEVOTO SH, MELANÇON E, EISEN JS, WESTERFIELD M (1996). Identification of separate slow and fast muscle precursor cells in vivo, prior to somite formation. Development 122: 3371-3380.

DUBOC V, LOGAN MPO (2011). Pitx1 is necessary for normal initiation of hindlimb outgrowth through regulation of Tbx4 expression and shapes hindlimb morphologies via targeted growth control. Development 138: 5301-5309.

DWORAK HA, CHARLES MA, PELLERANO LB, SINK H (2001). Characterization of Drosophilahibris, a gene related to human nephrin. Development 128:4265-4276.

EDOM-VOVARD F, SCHULER B, BONNIN M-A, TEILLET M-A, DUPREZ D (2002). Fgf4 Positively Regulates scleraxis and Tenascin Expression in Chick Limb Tendons. Dev Biol 247: 351-366.

FIELD, LH, MATHESON T (1998). Chrdotonal organs of insects. Advances in Insect Physiology, Volume 27, Academic Press Inc, San Diego (1998), pp. 1-228

FROMMER G, VORBRUGGEN G, PASCA G, JACKLE H, VOLKT (1996). Epidermal egr-like zinc finger protein of Drosophila participates in myotube guidance. EMBO J 15: 1642-1649.

GEORGE EL, GEORGES-LABOUESSE EN, PATEL-KING RS, RAYBURNH, HYNES $R O$ (1993). Defects in mesoderm, neural tube and vascular development in mouse embryos lacking fibronectin. Development 119: 1079-1091.

GILSOHN E, VOLK TA (2010a). screen for tendon-specific genes uncovers new and old components involved in muscle-tendon interaction. Fly (Austin) 4: 149-153.

GILSOHN E, VOLK T (2010b). Slowdown promotes muscle integrity by modulating integrin-mediated adhesion at the myotendinous junction. Development 137: 785-794.

GREENBLATT BEN-EL RT, HASSAN A, SALZBERG A (2017). Loss of thrombospondin reveals a possible role for the extracellular matrix in chordotonal cap cell elongation. Int J Dev Biol. doi:10.1387/ijdb.160275as

GREIG S, AKAM M (1993). Homeotic genes autonomously specify one aspect of pattern in the Drosophila mesoderm. Nature 362: 630-632.

GUERQUIN M-J, CHARVET B, NOURISSAT G, HAVIS E, RONSIN O, BONNIN M-A, RUGGIU M, OLIVERA-MARTINEZ I, ROBERT N, LU Y, KADLER KE, BAUMBERGER T, DOURSOUNIAN L, BERENBAUM F, DUPREZ D (2013). Transcription factor EGR1 directs tendon differentiation and promotes tendon repair. J Clin Invest 123: 3564-3576.

HALACHMIN, NACHMANA, SALZBERGA(2016). Anewly identified type of attachment cell is critical for normal patterning of chordotonal neurons. Dev Biol 411: 61-71.

HASSONP, DELAURIERA, BENNETTM, GRIGORIEVAE, NAICHE LA, PAPAIOANNOU VE, MOHUN TJ, LOGAN MPO (2010). Tbx4 and tbx5 acting in connective tissue are required for limb muscle and tendon patterning. Dev Cell 18: 148-156.

HIPPENMEYER S, SHNEIDER NA, BIRCHMEIER C, BURDEN SJ, JESSELL TM, ARBER S (2002). A role for neuregulin1 signaling in muscle spindle differentiation. Neuron 36: 1035-1049.

HUANGAH, RIORDANTJ, PRYCEB, WEIBELJL, WATSONSS, LONG F, LEFEBVRE V, HARFE BD, STADLER HS, AKIYAMA H, TUFA SF, KEENE DR, SCHWEITZER $R$ (2015). Musculoskeletal integration at the wrist underlies the modular development of limb tendons. Development 142: 2431-2441.

INBALA, LEVANON D, SALZBERG A (2003). Multiple roles for u-turn/ventral veinless in the development of Drosophila PNS. Development 130: 2467-2478.

INBALA, VOLK T, SALZBERG A (2004). Recruitment of ectodermal attachment cells via an EGFR-dependent mechanism during the organogenesis of Drosophila proprioceptors. Dev Cell 7: 241-250.

JAMI L (1992). Golgi tendon organs in mammalian skeletal muscle: functional properties and central actions. Physiol Rev 72: 623-666.

JENKINS MH, ALROWAISHED SS, GOODY MF, CRAWFORD BD, HENRY CA (2016). Laminin and Matrix metalloproteinase 11 regulate Fibronectin levels in the zebrafish myotendinous junction. Skelet Muscle 6: 18.

KARDON G (1998). Muscle and tendon morphogenesis in the avian hind limb. Development 125: 4019-4032.

KELEMAN K, DICKSON BJ (2001). Short- and Long-Range Repulsion by the Drosophila Unc5 Netrin Receptor. Neuron 32: 605-617.

KLEIN Y, HALACHMI N, EGOZ-MATIA N, TODER M, SALZBERG A (2010). The proprioceptive and contractile systems in Drosophila are both patterned by the EGR family transcription factor Stripe. Dev Biol 337: 458-470.

KRAFT-SHELEG O, ZAFFRYAR-EILOT S, GENIN O, YASEEN W, SOUEIDBAUMGARTENS, KESSLERO, SMOLKINT, AKIRI G, NEUFELD G, CINNAMON Y, HASSON P (2016). Localized LoxL3-Dependent Fibronectin Oxidation Regulates Myofiber Stretch and Integrin-Mediated Adhesion. Dev Cell 36: 550-561.

KRAUT R, ZINN K (2004). Roundabout 2 regulates migration of sensory neurons by signaling in trans. Curr Biol 14: 1319-1329.

KRZEMIEN J, FABRE CCG, CASAL J, LAWRENCE PA (2012). The muscle pattern of the Drosophila abdomen depends on a subdivision of the anterior compartment of each segment. Development 139: 75-83.

LEJARD V, BLAIS F, GUERQUIN M-J, BONNET A, BONNIN M-A, HAVIS E, MALBOUYRES M, BIDAUD CB, MARO G, GILARDI-HEBENSTREIT P, ROSSERT J, RUGGIERO F, DUPREZ D (2011). EGR1 and EGR2 involvement in vertebrate tendon differentiation. J Biol Chem 286: 5855-5867.

LU HH, THOMOPOULOS S (2013). Functional attachment of soft tissues to bone: development, healing, and tissue engineering. Annu Rev Biomed Eng 15:201-226.

MURCHISON ND, PRICE BA, CONNER DA, KEENE DR, OLSON EN, TABIN CJ, SCHWEITZER R (2007). Regulation of tendon differentiation by scleraxis distinguishes force-transmitting tendons from muscle-anchoring tendons. Development 134: 2697-708.

NABEL-ROSEN H, DOREVITCH N, REUVENY A, VOLK T (1999). The Balance between Two Isoforms of the Drosophila RNA-Binding Protein How Controls Tendon Cell Differentiation. Mol Cell 4: 573-584.

NABEL-ROSENH, VOLOHONSKY G, REUVENYA, ZAIDEL-BARR, VOLKT (2002) Two isoforms of the Drosophila RNA binding protein, how, act in opposing directions to regulate tendon cell differentiation. Dev Cell 2: 183-193.

NIR R, GROSSMAN R, PAROUSH Z, VOLK T (2012). Phosphorylation of the Drosophila melanogaster RNA-binding protein HOW by MAPK/ERK enhances its dimerization and activity. PLoS Genet 8: e1002632.

OLIVEIRA FERNANDES M, TOURTELLOTTE WG (2015). Egr3-dependent muscle spindle stretch receptor intrafusal muscle fiber differentiation and fusimotor in- 
nervation homeostasis. J Neurosci 35: 5566-5578.

ORDAN E, BRANKATSCHK M, DICKSON B, SCHNORRER F, VOLK T (2015). Slit cleavage is essential for producing an active, stable, non-diffusible short-range signal that guides muscle migration. Development 142: 1431-1436.

ORDAN E, VOLK T (2015a). Cleaved Slit directs embryonic muscles. Fly (Austin) 9: 82-85.

ORDAN E, VOLK T (2015b). A non-signaling role of Robo2 in tendons is essential for Slit processing and muscle patterning. Development 142: 3512-3518.

POLIAKS, NOROVICHAL, YAMAGATAM, SANES JR, JESSELLTM (2016). Muscletype Identity of Proprioceptors Specified by Spatially Restricted Signals from Limb Mesenchyme. Cell 164: 512-525.

SCHEJTER ED, BAYLIES MK (2010). Born to run: creating the muscle fiber. Curr Opin Cell Biol 22: 566-574.

SCHNORRER F, DICKSON BJ (2004). Muscle Building: Mechanisms of Myotube Guidance and Attachment Site Selection. Dev Cell 7: 9-20.

SCHWEITZER R, CHYUNG JH, MURTAUGH LC, BRENT AE, ROSEN V, OLSON EN, LASSARA, TABIN CJ (2001). Analysis of the tendon cell fate using Scleraxis, a specific marker for tendons and ligaments. Development 128: 3855-3866

SCHWEITZER R, ZELZER E, VOLK T (2010). Connecting muscles to tendons: tendons and musculoskeletal development in flies and vertebrates. Development 137: 2807-2817.

SNOW CJ, HENRY CA (2009). Dynamic formation of microenvironments at the myotendinous junction correlates with muscle fiber morphogenesis in zebrafish. Gene Expr Patterns 9: 37-42.

SNOW CJ, PETERSON MT, KHALIL A, HENRY CA (2008). Muscle development is disrupted in zebrafish embryos deficient for fibronectin. Dev Dyn 237: 2542-2553.

STEIGEMANN P, MOLITOR A, FELLERT S, JÄCKLE H, VORBRÜGGEN G (2004). Heparan Sulfate Proteoglycan Syndecan Promotes Axonal and Myotube Guidance by Slit/Robo Signaling. Curr Biol 14: 225-230.

SUBRAMANIAN A, PROKOP A, YAMAMOTO M, SUGIMURA K, UEMURA T, BETSCHINGER J, KNOBLICH JA, VOLK T (2003). Shortstop recruits EB1/
APC1 and promotes microtubule assembly at the muscle-tendon junction. Curr Biol 13: 1086-1095.

SUBRAMANIAN A, SCHILLING TF (2014). Thrombospondin-4 controls matrix assembly during development and repair of myotendinous junctions. Elife 3: e02372.

SUBRAMANIANA, SCHILLING TF (2015). Tendon development and musculoskeleta assembly: emerging roles for the extracellular matrix. Development142:4191-4204.

SUBRAMANIAN A, WAYBURN B, BUNCH T, VOLK T (2007). Thrombospondinmediated adhesion is essential for the formation of the myotendinous junction in Drosophila. Development 134: 1269-1278.

USUI K, PISTILLO D, SIMPSON P (2004). Mutual exclusion of sensory bristles and tendons on the notum of dipteran flies. Curr Biol 14: 1047-1055.

VOLK T, VIJAYRAGHAVAN K (1994). A central role for epidermal segment border cells in the induction of muscle patterning in the Drosophila embryo. Development 120: 59-70.

VOLOHONSKY G, EDENFELD G, KLAMBT C, VOLK T (2007). Muscle-dependent maturation of tendon cells is induced by post-transcriptional regulation of stripeA. Development 134: 347-356.

WANG S, REUVENYA, VOLKT (2015). Nesprin provides elastic properties to muscle nuclei by cooperating with spectraplakin and EB1. J Cell Biol 209: 529-538.

WAYBURN B, VOLK T (2009). LRT, a tendon-specific leucine-rich repeat protein promotes muscle-tendon targeting through its interaction with Robo. Development 136: 3607-3615.

WEITKUNAT M, KAYA-COPUR A., GRILL S W, SCHNORRER F (2014). Tension and force-resistant attachment are essential for myofibrillogenesis in Drosophila flight muscle. Curr Biol 24: 705-716.

YARNITZKY T, MIN L, VOLK T (1997). The Drosophila neuregulin homolog Vein mediates inductive interactions between myotubes and their epidermal attachment cells. Genes Dev 11: 2691-2700.

ZELZER E, BLITZ E, KILLIAN ML, THOMOPOULOS S (2014). Tendon-to-bone attachment: from development to maturity. Birth Defects Res C Embryo Today 102: 101-112. 


\section{Further Related Reading, published previously in the Int. J. Dev. Biol.}

Models of amphibian myogenesis - the case of Bombina variegata

Leokadia Kiełbwna and Marta Migocka-Patrzałek

Int. J. Dev. Biol. (2017) 61: 17-27

http://www.intjdevbiol.com/web/paper/160370mm

Selective depolarization of transmembrane potential alters muscle patterning and muscle cell localization in Xenopus laevis embryos Maria Lobikin, Jean-François Paré, David L. Kaplan and Michael Levin

Int. J. Dev. Biol. (2015) 59: 303-311

http://www.intjdevbiol.com/web/paper/150198ml

SDF-1 controls the muscle and blood vessel formation of the somite

Aisha Abduelmula Ruijin HuangQin Pu, Hirokazu Tamamura, Gabriela Morosan-Puopolo and Beate Brand-Saberi

Int. J. Dev. Biol. (2016) 60: 29-38

http://www.intjdevbiol.com/web/paper/150132rh

Sarcosin (Krp1) in skeletal muscle differentiation: gene expression profiling and knockdown experiments Leonie du Puy, Abdelaziz Beqqali, Helena T.A. van Tol, Jantine Monshouwer-Kloots, Robert Passier, Henk P. Haagsman and Bernard A.J. Roelen Int. J. Dev. Biol. (2012) 56: 301-309

http://www.intjdevbiol.com/web/paper/113327lp

Cytoskeletal heart-enriched actin-associated protein (CHAP) is expressed in striated and smooth muscle cells in chick and mouse during embryonic and adult stages

Willemijn van Eldik, Abdelaziz Beqqali, Jantine Monshouwer-Kloots, Christine Mummery and Robert Passier

Int. J. Dev. Biol. (2011) 55: 649-655

http://www.intjdevbiol.com/web/paper/103207wv

Visualization, characterization and modulation of calcium signaling during the development of slow muscle cells in intact zebrafish embryos

Chris Y. Cheung, Sarah E. Webb, Donald R. Love and Andrew L. Miller

Int. J. Dev. Biol. (2011) 55: 153-174

http://www.intjdevbiol.com/web/paper/103160cc

Limb muscle development

Bodo Christ and Beate Brand-Saberi

Int. J. Dev. Biol. (2002) 46: 905-914

http://www.intjdevbiol.com/web/paper/12455628

A dynamic requirement for community interactions during Xenopus myogenesis Henrietta J Standley, Aaron M Zorn and John B Gurdon

Int. J. Dev. Biol. (2002) 46: 279-283

http://www.intjdevbiol.com/web/paper/12068948
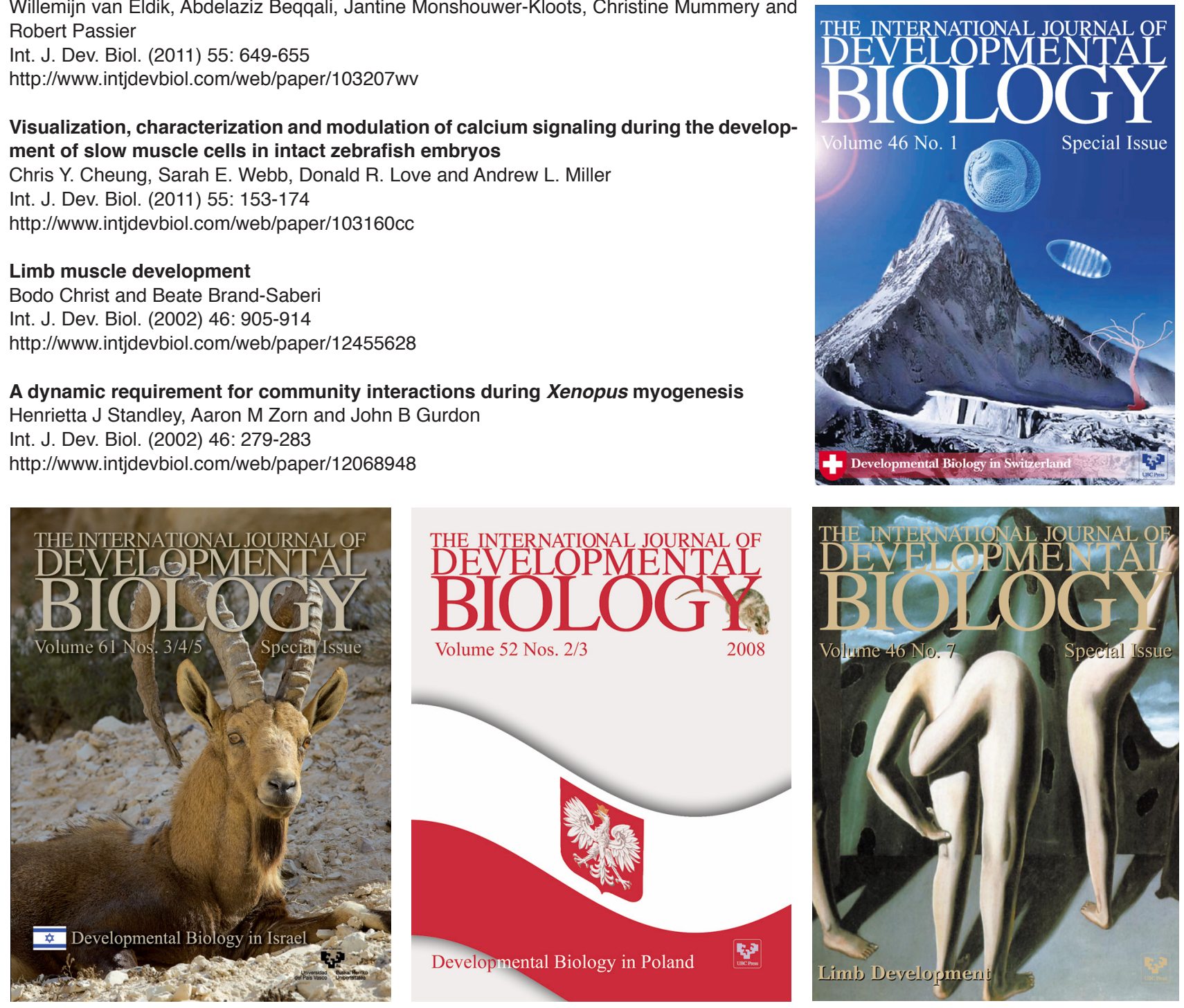